\title{
Static Scheduling of Monoprocessor Real-Time Systems composed of Hard and Soft Tasks
}

\author{
Luis Alejandro Cortés, Petru Eles, Zebo Peng \\ Department of Computer and Information Science \\ Linköping University, S-581 83 Linköping, Sweden \\ \{luico,petel,zebpe\}@ida.liu.se
}

\begin{abstract}
In this paper we address the problem of static scheduling of real-time systems that include both hard and soft tasks. We consider that hard as well as soft tasks are periodic and that there exist data dependencies among tasks. In order to capture the relative importance of soft tasks and how the quality of results is affected when missing a soft deadline, we use utility functions associated to soft tasks. Thus our objective is to find an execution order for tasks that maximizes the total utility and at the same time guarantees hard deadlines. We use the expected duration of tasks for evaluating utility functions whereas we use the maximum duration of tasks for ensuring that hard deadlines are always met. We present an algorithm for finding the optimal schedule and also different heuristics that find near-optimal solutions at reasonable computational cost. The proposed algorithms are evaluated using a large number of synthetic examples.
\end{abstract}

\section{Introduction}

There exist classes of real-time systems that require the execution of tasks which have distinct types of timing constraints. Such systems include activities whose completion before a given deadline is critical to the overall behavior of the system. Missing one such deadline has severe or catastrophic consequences, hence these tasks are referred to as hard. At the same time, these real-time systems include activities that have looser timing constraints and a deadline miss can be tolerated though the quality of results might degrade. Such tasks are referred to as soft.

The problem of jointly scheduling hard and soft tasks has been studied, for example, in the frame of integrating multimedia applications into hard real-time systems [5], [1].

Most of the approaches consider that hard tasks are periodic whereas soft tasks are aperiodic. Both dynamic [2], [8] and fixed priority systems [4], [6] have been considered, aiming to minimize in both cases the response time of soft aperiodic tasks. These, as well as most of previous work, assume that the sooner a soft task is served the better but make no distinction among soft tasks, that is, there is no relative importance of soft tasks.

In this paper we address the problem of static scheduling (at design-time) of real time-systems made up of hard and soft tasks. We consider that both hard and soft tasks are periodic and, in order to capture the significance of soft tasks, we make use of utility functions (value or utility functions were first suggested by Locke [7] to represent importance and criticality of tasks). As opposed to the approaches cited above where tasks are assumed independent, we take into account the precedence relation among tasks.

Most of earlier work uses only the worst case execution time (WCET) for scheduling both hard and soft tasks (Abeni and Buttazzo's approach [1] does use WCET for guaranteeing hard deadlines and mean values for serving soft tasks though). We consider the fact that the actual execution time of a task is rarely its WCET. Thus we use the expected duration of tasks when evaluating the utility functions associated to soft tasks (we aim to find the schedule for which the total utility is maximum) and we use the maximum duration of tasks for ensuring that all hard deadlines are met in every possible scenario.

\section{Preliminaries}

We consider that the system is represented by a directed acyclic graph $G=(T, E)$ where its nodes correspond to tasks and their data dependencies are given by the graph edges. Throughout this paper we assume that all the tasks of the system are mapped into a single processor.

We use ${ }^{\circ} t$ to denote set of the predecessors of task $t$, that is, ${ }^{\circ} t=\left\{t^{\prime} \in T \mid\left\langle t^{\prime}, t\right\rangle \in E\right\}$. Similarly, $t^{\circ}=\left\{t^{\prime} \in T \mid\right.$ $\left.\left\langle t, t^{\prime}\right\rangle \in E\right\}$ denotes the set of successors of task $t$.

The actual execution time of every task $t \in T$ at a certain activation of the system, denoted $|t|$, lies in the interval bounded by the minimum duration $l(t)$ and the maximum duration $m(t)$ of the task, i.e. $l(t) \leq|t| \leq m(t)$. In our analysis we take into consideration the expected duration $e(t)$ of every task $t \in T$, which is the mean value of the possible execution times of the task. In the simple case that the execution time is uniformly distributed over the interval $[l(t), m(t)]$, we have $e(t)=(l(t)+m(t)) / 2$. For an arbitrary continuous probability distribution $f(\tau)$, the expected duration is $e(t)=\int_{l(t)}^{m(t)} \tau f(\tau) d \tau$.

We define a schedule as the execution order for the tasks in the system. We assume a single-rate semantics, that is, each task is executed exactly once for every activation of the system. Thus a schedule is a bijection $\sigma: T \rightarrow\{1,2, \ldots,|T|\}$. We use $\sigma=t_{1} t_{2} \ldots t_{n}$ as shorthand for $\sigma\left(t_{1}\right)=1, \sigma\left(t_{2}\right)=$ $2, \ldots, \sigma\left(t_{n}\right)=|T|$. We assume that the system is activated periodically. Handling tasks with different periods is possible by generating several instances of the tasks and building a graph that corresponds to a set of tasks as they occur within a time period that is equal the least common multiple of the periods of the involved tasks.

In this context, a schedule does not provide the starting time for tasks, only their execution sequence. Thus, for the schedule $\sigma=t_{1} t_{2} \ldots t_{n}$, task $t_{1}$ will start when the system is activated and task $t_{i+1}, 1 \leq i<n$, will start executing as soon as task $t_{i}$ has finished. For a given schedule, the completion time of a task $t_{i}$ is denoted $\tau_{i}$. In the sequel, the starting and completion times that we use are relative to the system activation instant. For example, for the schedule $\sigma=$ $t_{1} t_{2} \ldots t_{n}, t_{1}$ starts executing at time 0 and its completion time is $\tau_{1}=\left|t_{1}\right|$, the completion time of $t_{2}$ is $\tau_{2}=\tau_{1}+\left|t_{2}\right|$, and so forth.

The tasks that make up a system can be classified as non-real-time, hard, or soft. Non-real-time tasks are neither hard nor soft, and have no timing constraints, though they may influence other hard or soft tasks through precedence constraints as defined by the task graph $G=(T, E)$. Both hard and soft tasks have deadlines. A hard deadline $d(h)$ is the time by which a hard task $h \in T$ must be completed, 
otherwise the integrity of the system is jeopardized. A soft deadline $d(s)$ is the time by which a soft task $s \in T$ should be completed. Lateness of soft tasks is acceptable though it decreases the quality of results. In order to capture the relative importance among soft tasks and how the quality of results is affected when missing a soft deadline, we use a non-increasing utility function $u_{j}\left(\tau_{j}\right)$ for each soft task $s_{j}$. Typical utility functions are depicted in Fig. 1.
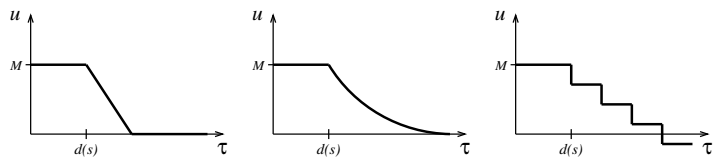

Fig. 1. Typical utility functions for soft tasks

In this paper we address the problem of finding a schedule that maximizes the sum of individual utilities of soft tasks when considering expected execution times, yet guaranteeing that hard deadlines are always met. Such a sum is called total utility and denoted $U\left(U=\sum_{s_{j} \in S} u_{j}\left(\tau_{j}\right)\right.$, where $S$ is the set of soft tasks).

\section{Motivational Example}

Let us consider a system that has five tasks $t_{1}, t_{2}, t_{3}, t_{4}$, and $t_{5}$, with data dependencies as shown in the graph of Fig. 2. The expected and maximum duration of every task are given in Fig. 2 in the form $e_{i}=e\left(t_{i}\right)$ and $m_{i}=m\left(t_{i}\right)$ respectively. The only hard task in the system is $t_{4}$ and its deadline is $d\left(t_{4}\right)=30$. Tasks $t_{2}$ and $t_{3}$ are soft, their deadlines are $d\left(t_{2}\right)=9$ and $d\left(t_{3}\right)=21$, and their utility functions are given in Fig. 2.

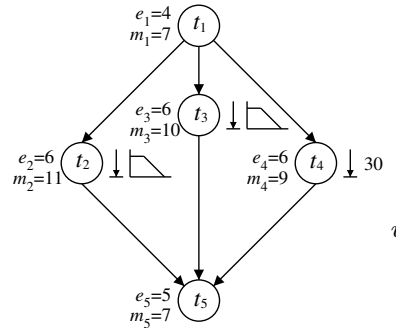

$$
\begin{gathered}
u_{2}\left(\tau_{2}\right)= \begin{cases}3 & \text { if } \tau_{2} \leq 9, \\
\frac{9}{2}-\frac{\tau_{2}}{6} & \text { if } 9 \leq \tau_{2} \leq 27, \\
0 & \text { if } \tau_{2} \geq 27 .\end{cases} \\
u_{3}\left(\tau_{3}\right)= \begin{cases}2 & \text { if } \tau_{3} \leq 21, \\
16-\frac{2 \tau_{3}}{3} & \text { if } 21 \leq \tau_{3} \leq 24, \\
0 & \text { if } \tau_{3} \geq 24 .\end{cases}
\end{gathered}
$$

Fig. 2. Motivational example

When we consider the expected duration for every task, that is $|t|=e(t)$ for each $t \in T$, the schedule $\sigma_{a}=t_{1} t_{2} t_{3} t_{4} t_{5}$ is the one that maximizes the total utility: the completion times for tasks $t_{2}$ and $t_{3}$ are $\tau_{2}=10$ and $\tau_{3}=16$, and the total utility is $U_{a}=u_{2}(10)+u_{3}(16)=17 / 6+2 \approx 4.83$. However, $\sigma_{a}$ does not guarantee the satisfaction of hard deadlines (take the possible scenario where $\left|t_{1}\right|=7,\left|t_{2}\right|=\left|t_{3}\right|=10$, $\left|t_{4}\right|=8$ : in such a case $\tau_{4}=35$ and therefore $t_{4}$ misses its deadline).

We aim to find the schedule that maximizes the total utility (sum of individual contributions by soft tasks), while guaranteeing that hard deadlines are met in all possible scenarios. When we consider only the upper bounds of execution time for every task, that is $|t|=m(t)$ for each $t \in T$, we obtain the schedule $\sigma_{b}=t_{1} t_{3} t_{4} t_{2} t_{5}$ which maximizes the total utility when every task takes its maximum duration and, at the same time, guarantees no hard deadline miss.

Although $\sigma_{b}$ ensures that hard deadlines are always satisfied, it gives the maximum utility in the particular case of
WCET for all tasks, a situation that, though possible, seldom occurs. It is better to find the schedule that yields the maximum utility in the more likely case of expected duration for all tasks, yet guaranteeing no hard deadline miss. Thus we must obtain the schedule that guarantees meeting all hard deadlines, when maximum duration is considered, and maximizes the total utility, when expected duration is considered. Such a schedule for the example of Fig. 2 is $\sigma_{c}=t_{1} t_{2} t_{4} t_{3} t_{5}$. When every task lasts its expected duration, following $\sigma_{c}, t_{2}$ completes at $\tau_{2}=10$ and $t_{3}$ completes at $\tau_{3}=22$, and thus the total utility is $U_{c}=u_{2}(10)+u_{3}(22)=17 / 6+4 / 3 \approx 4.17$. Note that in the same case (expected duration for all tasks) $\sigma_{b}=t_{1} t_{3} t_{4} t_{2} t_{5}$ yields a utility $U_{b}=u_{2}(22)+u_{3}(10)=5 / 6+2 \approx 2.83$.

\section{Problem Formulation}

We have informally described the problem of scheduling real-time systems that have both soft and hard tasks. We want to find the schedule (an execution sequence for tasks) that, among all schedules that respect the hard constraints in the worst-case, maximizes the total utility when tasks last their expected duration.

The formulation of Scheduling WITH SOFT AND HARD Tasks to Maximize Utility (SSHMU) is as follows. Given - a set $T$ of tasks,

- a directed acyclic graph $G=(T, E)$ defining precedence constraints for the tasks,

- a maximum duration $m(t) \in \mathbb{N}$ for each task $t \in T$,

- an expected duration $e(t) \in \mathbb{N}$ for each task $t \in T$ $(e(t) \leq m(t))$

- a subset $H \subseteq T$ of hard tasks,

- a deadline $d(h) \in \mathbb{N}$ for each hard task $h \in H$,

- a subset $S \subseteq T$ of soft tasks $(S \cap H=\emptyset)$, and

- a non-increasing utility function $u_{j}\left(\tau_{j}\right)$ for each soft task $s_{j} \in S\left(\tau_{j}\right.$ is the completion time of $\left.s_{j}\right)$;

find a one-processor schedule $\sigma$ (a bijection $\sigma: T \rightarrow$ $\{1,2, \ldots,|T|\})$ that maximizes

$$
\sum_{s_{j} \in S} u_{j}\left(\tau_{j}^{e}\right)
$$

where $\tau_{j}^{e}$ is the expected completion time ${ }^{1}$ of task $s_{j}$, subject to:

- $\sigma(t)<\sigma\left(t^{\prime}\right)$ for all $\left\langle t, t^{\prime}\right\rangle \in E$, and

- $\tau_{i}^{m} \leq d\left(h_{i}\right)$ for all $h_{i} \in H$, where $\tau_{i}^{m}$ is the maximum completion time ${ }^{2}$ of task $h_{i}$.

\section{Exact Algorithm}

It has been proved that Scheduling WiTH Soft AND Hard Tasks to Maximize UtiLity is an NP-complete problem [3]. Therefore, unless $\mathbf{N P}=\mathbf{P}$, there is no algorithm that solves every instance of the problem in polynomial time. This section presents an exact algorithm for SSHMU whose time complexity is $O\left(|T|^{3}|H||S| !\right)$.

The algorithm presented in Fig. 3 computes the schedule that maximizes the total utility when tasks last their expected duration, while guaranteeing that all hard deadlines

$$
\begin{gathered}
{ }^{1} \tau_{j}^{e} \text { is given by } \\
\tau_{j}^{e}= \begin{cases}e\left(t_{j}\right) & \text { if } \sigma\left(t_{j}\right)=1, \\
\tau_{k}^{e}+e\left(t_{j}\right) & \text { if } \sigma\left(t_{j}\right)=\sigma\left(t_{k}\right)+1 .\end{cases} \\
{ }^{2} \tau_{i}^{m} \text { is given by } \\
\tau_{i}^{m}= \begin{cases}m\left(t_{i}\right) & \text { if } \sigma\left(t_{i}\right)=1, \\
\tau_{k}^{m}+m\left(t_{i}\right) & \text { if } \sigma\left(t_{i}\right)=\sigma\left(t_{k}\right)+1 .\end{cases}
\end{gathered}
$$


are met even when all tasks last their maximum duration. Initially we check, by using the algorithm IsSCHEDULABLE, whether there exists at all a schedule that satisfies the hard time constraints. For each one of the possible permutations $S_{k}$ of soft tasks, the algorithm first checks whether $S_{k}$ is valid (that is, the order given by $S_{k}$ does not violate data dependencies) through the procedure IsVALIDPERM(S) which just checks whether there exists a path from the soft task $S_{[j]}$ to the soft task $\mathbf{S}_{[i]}$, for $j>i$ : if so, $\mathbf{S}$ is not valid. If $\mathbf{S}_{k}$ is valid, the algorithm OptimalSCHEDULE computes the best schedule $\sigma_{k}$ (the one that yields the highest total utility) for the particular order for soft tasks as expressed by $S_{k}$. The schedule $\sigma$ that, among all $\sigma_{k}$, provides the highest total utility when considering the expected duration for all tasks is the optimal one.

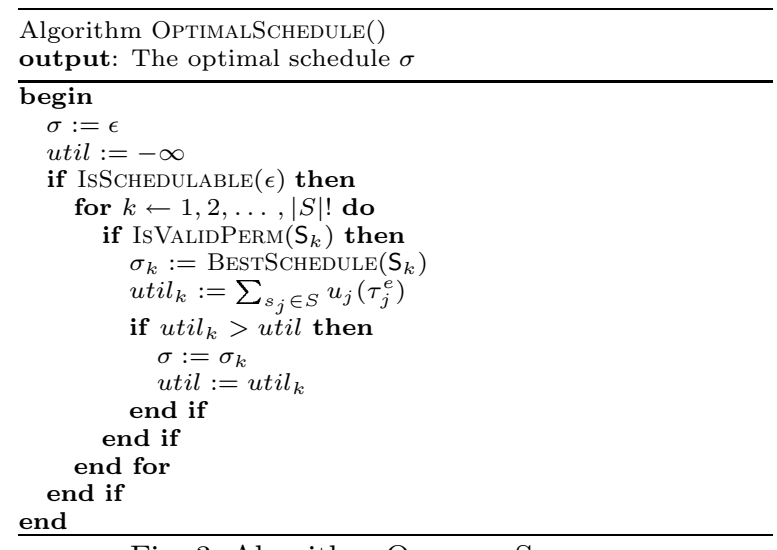

Fig. 3. Algorithm OptimalSchedule

The algorithm that computes the best schedule, for a given permutation of soft tasks $\mathrm{S}$, is presented in Fig. 4. The rationale is that the maximum total utility for the particular permutation $S$ is obtained when the soft tasks are set in the schedule as early as possible respecting the order given by $\mathrm{S}$. Let us consider again the example given in Fig. 2. There are two permutations of soft tasks $\mathrm{S}_{1}=\left[t_{2}, t_{3}\right]$ and $\mathrm{S}_{2}=\left[t_{3}, t_{2}\right]$. The schedules that obey the order for soft tasks given by the permutation $S_{1}$ (and also the precedence constraints imposed by the task graph) are $\sigma_{1}=t_{1} t_{2} t_{4} t_{3} t_{5}, \sigma_{1}^{\prime}=t_{1} t_{4} t_{2} t_{3} t_{5}$, and $\sigma_{1}^{\prime \prime}=t_{1} t_{2} t_{3} t_{4} t_{5}$. Note, first of all, that $\sigma_{1}^{\prime \prime}$ implies potential hard deadlines misses and therefore cannot be considered. Both $\sigma_{1}$ and $\sigma_{1}^{\prime}$ guarantee that hard deadlines are always met but $\sigma_{1}$ is better from the perspective of higher total utility. $\sigma_{1}=t_{1} t_{2} t_{4} t_{3} t_{5}$ is the schedule that sets soft tasks as early as possible (guaranteeing hard deadlines) respecting the order given by $\mathrm{S}_{1}=\left[t_{2}, t_{3}\right]$.

A simple proof of the fact that by setting soft tasks as early as possible according to the order given by $\mathrm{S}$ we get the maximum total utility for $\mathbf{S}$ is as follows: let $\sigma$ be the schedule that respects the order of soft tasks given by $\mathrm{S}$ (that is, $\left.1 \leq i<j \leq|\mathrm{S}| \Rightarrow \sigma\left(\mathrm{S}_{[i]}\right)<\sigma\left(\mathrm{S}_{[j]}\right)\right)$ and such that soft tasks are set as early as possible (that is, for every schedule $\sigma^{\prime}$, different from $\sigma$, that obeys the order of soft tasks given by $\mathrm{S}$ and respects all hard deadlines in the worst-case, $\sigma^{\prime}\left(\mathrm{S}_{[i]}\right)>\sigma\left(\mathrm{S}_{[i]}\right)$ for some $\left.1 \leq i \leq|\mathrm{S}|\right)$. Take one such $\sigma^{\prime}$. For at least one soft task $s_{j} \in S$ it holds $\sigma^{\prime}\left(s_{j}\right)>\sigma\left(s_{j}\right)$, therefore $\tau_{j}^{\prime e}>\tau_{j}^{e}\left(\tau_{j}^{\prime e}\right.$ is the completion time of $s_{j}$ when we use $\sigma^{\prime}$ as schedule while $\tau_{j}^{e}$ is the completion time of $s_{j}$ when $\sigma$ is used as schedule, considering in both cases expected du- ration for all tasks). Thus $u_{j}\left({\tau_{j}^{\prime}}_{j}^{e}\right) \leq u_{j}\left(\tau_{j}^{e}\right)$ because utility functions for soft tasks are non-increasing. Consequently $U^{\prime} \leq U$, where $U^{\prime}$ and $U$ are the total utility when using, respectively, $\sigma^{\prime}$ and $\sigma$ as schedules. Hence we conclude that no schedule $\sigma^{\prime}$, which respects the order for soft tasks given by $\mathrm{S}$, will yield a total utility greater than the one by $\sigma$.

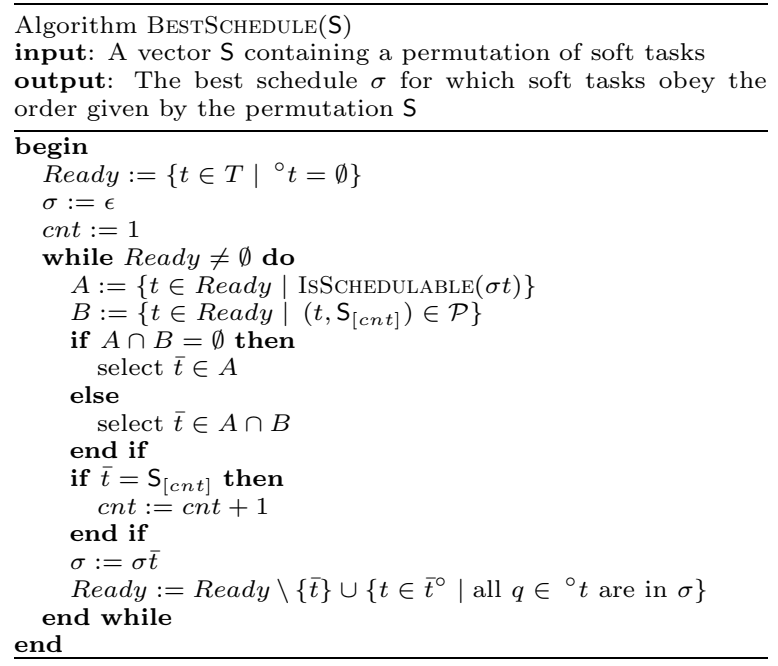

Fig. 4. Algorithm BestSchedule

The algorithm BeStSchedule(S) first tries to schedule the soft task $S_{[1]}$ as early as possible. In order to do so, it will set in first place all tasks from which there exists a path leading to $\mathrm{S}_{[1]}$, taking care of not incurring potential deadlines misses by the hard tasks. Then, a similar procedure is followed for $\mathrm{S}_{[2]}, \mathrm{S}_{[3]}, \ldots, \mathrm{S}_{[|S|]}$.

The algorithm BestSchedule(S) keeps a list Ready of tasks that are available at every step and constructs the schedule by progressively concatenating tasks to the string $\sigma$ (initially $\sigma=\epsilon$ ). In Fig. $4, A$ is the set of available tasks that, at that step, can be added to $\sigma$ without posing the risk of hard deadline misses. In other words, if we added a task $t \in \operatorname{Ready} \backslash A$ to $\sigma$ we could no longer guarantee that all hard constraints are met. $B$ is the set of available tasks that have a path to the next soft task $\mathrm{S}_{[c n t]}$ to be scheduled. $\mathcal{P}$ denotes the path relation $\left(\mathcal{P}=\left\{\left(t, t^{\prime}\right) \in T \times T \mid\right.\right.$ there is a path leading from $t$ to $\left.\left.t^{\prime}\right\}\right)$ and corresponds to the reflexive transitive closure of the relation $E$ (set of edges in the task graph), and it is computed only once for a given system. Once an available task $\bar{t}$ is selected, it is concatenated to $\sigma(\sigma:=\sigma \bar{t}), \bar{t}$ is removed from Ready, and all its successors that become available are added to Ready.

At every iteration of the while loop of the algorithm given in Fig. 4, we construct the set $A$ by checking, for every $t \in R e a d y$, whether concatenating $t$ to the schedule prefix $\sigma$ would imply a possible hard deadline miss. For this purpose we use the algorithm ISSCHEDULABLE( () , which returns a boolean indicating whether there is a schedule that agrees with the prefix $\varsigma$ and such that hard deadlines are met.

\section{Heuristics}

In this section we present several heuristic procedures for finding a near-optimal solution to the problem of scheduling with soft and hard tasks to maximize utility as formulated in Section 4. 
The algorithms progressively construct the schedule $\sigma$ by concatenating tasks to the string $\sigma$ that at the end will contain the final schedule. All the heuristics that we propose in this section make use of the list Ready of available tasks at every step. The heuristics differ in how the next task, among those in Ready, is selected as the one to be concatenated to $\sigma$. Note that the algorithms presented in this section are applicable only if the system is schedulable in first place (there exists a schedule that satisfies the hard time constraints).

The algorithms make use of a list scheduling heuristic. The basic algorithm is shown in Fig. 5. Initially, $\sigma=\epsilon$ (the empty string) and the list Ready contains those tasks that have no predecessor. The set $A$ contains the tasks that are in $\sigma$ (initially $A:=\emptyset$ ). The while loop is executed exactly $|T|$ times. At every iteration we compute the set $B$ of ready tasks that do not pose risk of hard deadline misses by being concatenated to the schedule prefix $\sigma$. If all soft tasks have already been set in $\sigma$ we select any $\bar{t} \in B$, else we compute a priority for soft tasks (SP $:=\operatorname{PRIORITY}(\sigma))$. The way such priorities are calculated is what differentiates the heuristics proposed in this paper. Among those soft tasks that are not in $\sigma$, we select $s_{k}$ as the one with the highest priority. Then, we compute the set $C$ of tasks that cause no hard deadline miss and that have a path leading to $s_{k}$. We select any $\bar{t} \in C$ if $C \neq \emptyset$, else we choose any $\bar{t} \in B$. Once an available task $\bar{t}$ is selected as described above, it is concatenated to $\sigma, \bar{t}$ is added to $A, \bar{t}$ is removed from the list Ready, and those successors of $\bar{t}$ that become available are added to Ready.

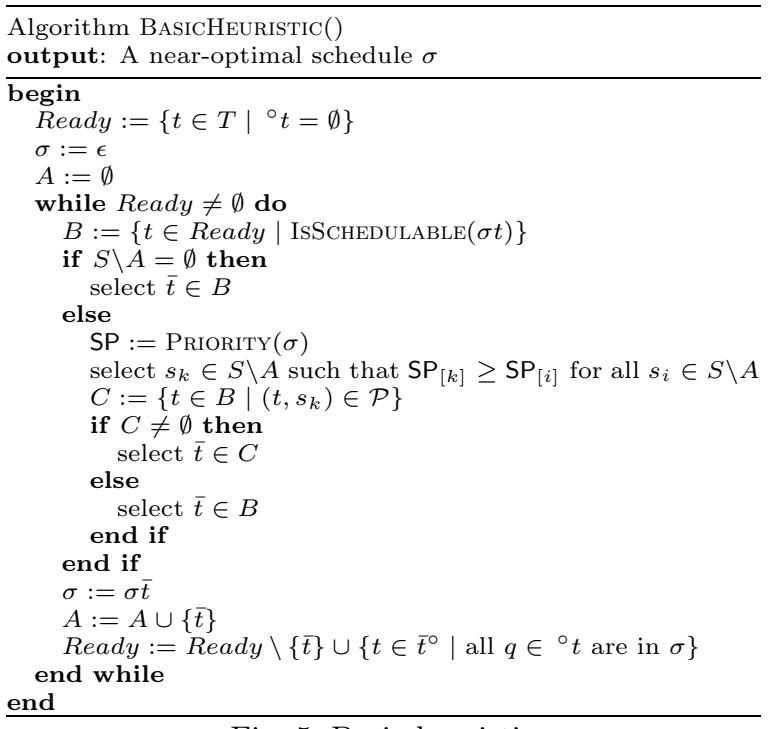

Fig. 5. Basic heuristic

The first of the proposed heuristics makes use of the basic algorithm presented in Fig. 5 and the algorithm given in Fig. 6 for computing the priorities of soft tasks. The procedure PRIORITYMAXUtiLity $(\varsigma)$ assigns a priority to soft tasks, for a given schedule prefix $\varsigma$, as follows: if $s_{i}$ is in $\varsigma$, its priority is $\mathrm{SP}_{[i]}:=-\infty$; if $s_{i}$ is not in $\varsigma$, we compute the earliest completion time $\tau_{i}^{e^{\prime}}$ when considering expected duration for all tasks. Then we make use of the maximum utility $M_{i}$ (see Fig. 1) for $s_{i}$ in order to calculate $\mathrm{SP}_{[i]}:=M_{i} / \tau_{i}^{e^{\prime}}$.

The algorithm PriorityMaxUtility makes use of $M_{i}=$ $u_{i}(0)$ for every soft task $s_{i} \in S$ but does not exploit the transition functions $u_{i}\left(\tau_{i}\right)$ themselves. Our second heuristic pro- cedure relies on the algorithm PRIORItySingleUtility $(\varsigma)$ (Fig. 7) for computing the priorities of soft tasks. If $s_{i}$ is in $\varsigma, \mathrm{SP}_{[i]}:=-\infty$, else we compute $\tau_{i}^{e^{\prime}}$ (it corresponds to the completion time, considering expected durations, of $s_{i}$ in a schedule that agrees with the prefix $\varsigma$ and for which $s_{i}$ is set the earliest). Then we assign the priority $\mathrm{SP}_{[i]}$ as the single utility of $s_{i}$ evaluated at $\tau_{i}^{e^{\prime}}$.

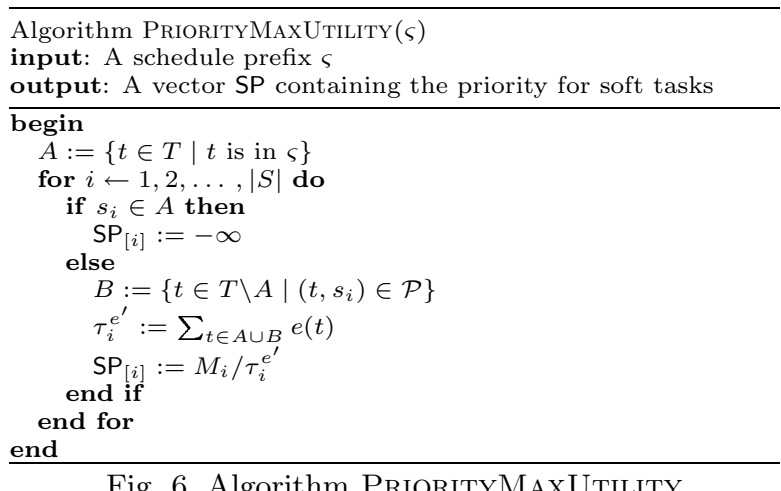

The algorithm PRIORITyTotalUtility $(\varsigma)$ shown in Fig. 8 also exploits the information of utility functions but, as opposed to PrioritySingleUtility, it considers the utility contributions of other soft tasks when computing the priority $\mathrm{SP}_{[i]}$ of the soft task $s_{i}$. If the soft task $s_{i}$ is not in $\varsigma$ its priority is computed as follows. First, we obtain the completion time $\tau_{i}^{e^{\prime}}$ when $s_{i}$ is earliest set in a schedule that agrees with the prefix $\varsigma$, using expected durations. Second, for each soft task $s_{j}$ different from $s_{i}$ that is not in $\varsigma$, we compute $\tau_{j}^{e^{\prime}}$ and $\tau_{j}^{e^{\prime \prime}}$. The former corresponds to the completion time when $s_{i}$ is earliest set in a schedule that agrees with the prefix $\varsigma$. The latter corresponds to the completion time when $s_{i}$ is latest set in a schedule that agrees with $\varsigma$. In both cases, expected duration of tasks are considered. The average of $\tau_{j}^{e^{\prime}}$ and $\tau_{j}^{e^{\prime \prime}}$ is used as argument for the utility function $u_{j}$. Thus the priority of $s_{i}$ is given by $\mathrm{SP}_{[i]}:=u_{i}\left(\tau_{i}^{e^{\prime}}\right)+\sum_{s_{j} \in S \backslash\left(A \cup\left\{s_{i}\right\}\right)} u_{j}\left(\left(\tau_{j}^{e^{\prime}}+\tau_{j}^{e^{\prime \prime}}\right) / 2\right)$.

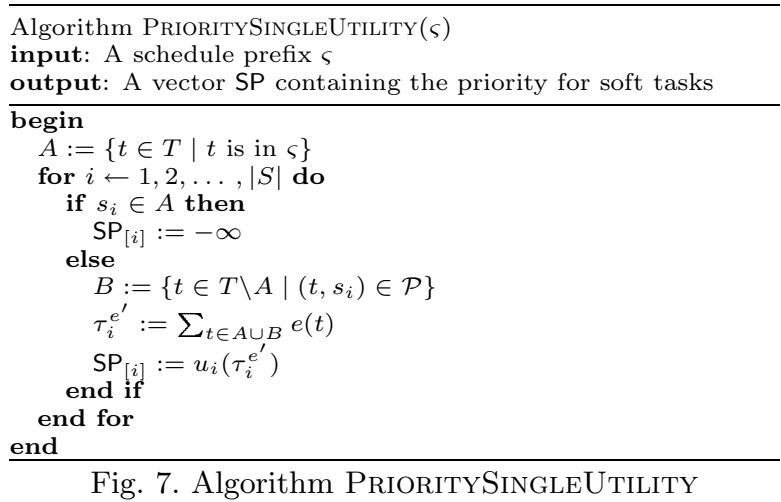

To sum up this section, we have presented three heuristics that are based on the algorithm of Fig. 5 and their difference lies in how the priorities for soft tasks are calculated. The first heuristic uses PriorityMaxUTILITy (Fig. 6), the second uses PrioritySingleUtility (Fig. 7), and the third one uses PriorityTotalUtility (Fig. 8). We have named the heuristics after the algorithms they use for computing 
priorities: MaxUtility (MU), SingleUtility (SU), and TotalUtility (TU), respectively. The first two have a time complexity $O\left(|T|^{3}(|H|+|S|)\right)$ whereas the third has a time complexity $O\left(|T|^{3}\left(|H|+|S|^{2}\right)\right)$.

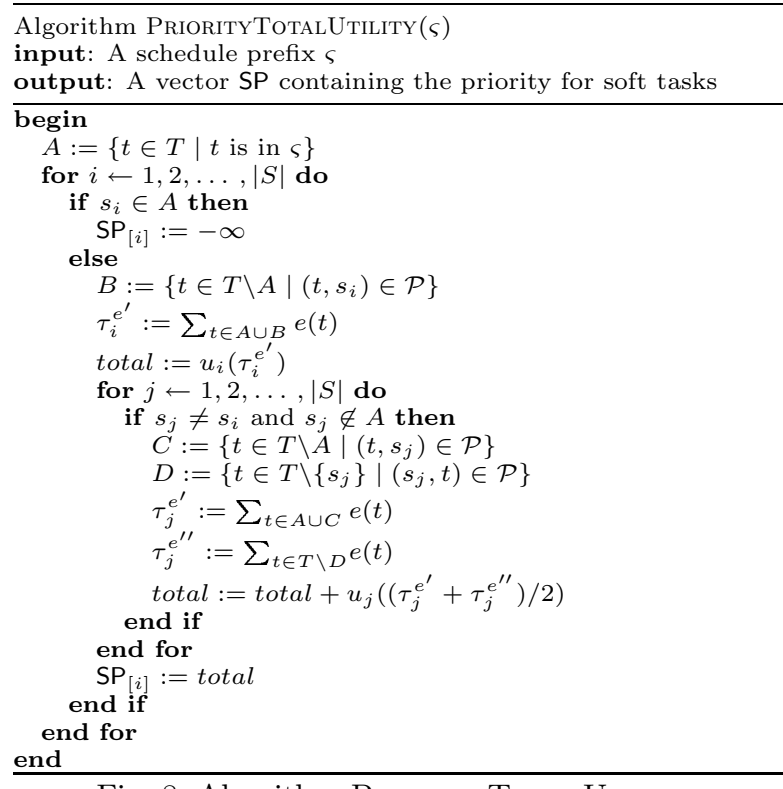

Fig. 8. Algorithm PriorityTotalUtility

\section{Experimental Results}

In this section we experimentally evaluate the heuristics proposed in Section 6. We are initially interested in the quality of the schedules obtained by the heuristics MaxUtiLity (MU), SingleUtility (SU), and TotalUtility (TU) with respect to the optimal schedule as given by the exact algorithm OptimalSCHEDULE. We use as criterion the deviation dev $=\left(U_{\text {opt }}-U_{\text {heur }}\right) / U_{\text {opt }}$ where $U_{\text {opt }}$ is the total utility given by the optimal schedule and $U_{\text {heur }}$ is the total utility corresponding to the schedule obtained with a heuristic.

We have randomly generated a large number of tasks graphs in our experiments. We initially considered graphs with 100, 200, 300, 400, 500, and 600 tasks. For these, we considered systems with 2, 3, 4, 5, 6, 7, and 8 soft tasks. For the case $|T|=200$ tasks, we considered systems with 25, 50, 75,100 , and 125 hard tasks. We generated 500 graphs for each graph dimension. Expected and maximum durations of tasks were also assigned randomly. For every task graph, hard tasks and their deadlines were selected randomly as well as soft tasks and their utility functions. All the experiments were run on a Sun Ultra 10 workstation.

We have plotted the average deviation as a function of the number of tasks in Figs. 9(a) and 9(b). These correspond to systems with 5 and 8 soft tasks respectively. All the systems considered in Figs. 9(a) and 9(b) have 50 hard tasks. These plots consistently show that heuristic TotalUtility (TU) gives the best results for the considered cases.

The plot in Fig. 10(a) depicts the average deviation as a function of the number of hard tasks. In this case, we have considered systems with 200 tasks, out of which 5 are soft. In this graph we observe that the number of hard tasks does not affect significantly the quality the schedules obtained with the proposed heuristics.
We have also studied the average deviation as a function of the number of soft tasks and the results are plotted in Fig. 10(b). The considered systems have 100 tasks, 50 of them being hard. We again see that the heuristic TU consistently provides the best results. We can also note that there is a trend showing an increasing average deviation as the number of soft tasks grows, especially for the heuristics MU and SU.

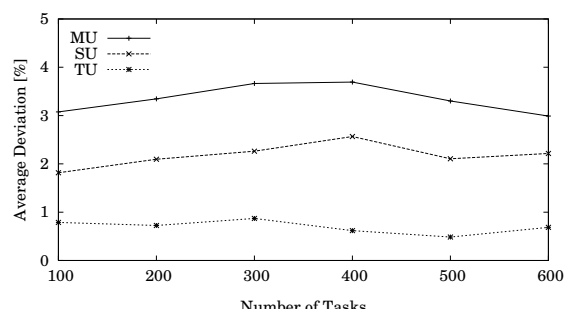

(a) 5 soft tasks

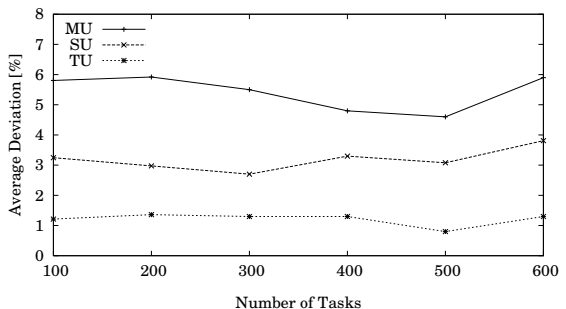

(b) 8 soft tasks

Fig. 9. Evaluation of the heuristics (50 hard tasks)

Note that, in the experiments we have presented so far, the number of soft tasks is small. Recall that the time complexity of the exact algorithm is $O\left(|T|^{3}|H||S| !\right)$ and therefore any comparison that requires computing the optimal schedule is infeasible for a large number of soft tasks.

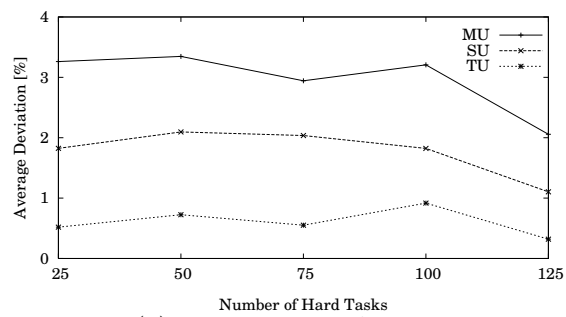

(a) 200 tasks, 5 soft tasks

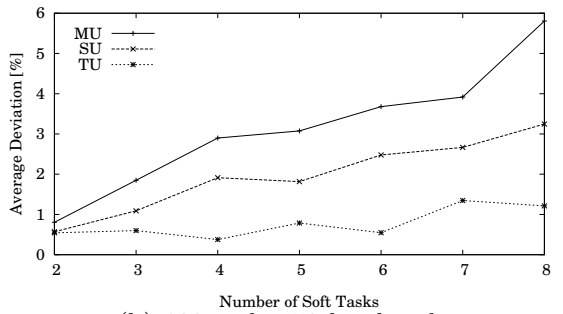

(b) 100 tasks, 50 hard tasks

Fig. 10. Evaluation of the heuristics

In a second set of experiments, we have compared the heuristics among themselves considering systems with larger numbers of soft and hard tasks. We normalize the utility obtained the heuristics with respect to the utility given by the algorithm TotalUtility (TU): $\left\|U_{\text {heur }}\right\|=U_{\text {heur }} / U_{T U}$.

We generated, for these experiments, graphs with 500 
tasks and considered cases with 50, 100, 150, 200, and 250 hard tasks and 50,100, 150, 200, and 250 soft tasks. The results are shown in Figs. 11(a) and 11(b). We can note that for systems with many soft tasks, the algorithm SingleUTILITY gives results very close to those of the algorithm TOTALUtility. In the particular case $T=500, H=150, S=200$, SU slightly outperforms TU $\left(\left\|U_{S U}\right\|=1.0014,\left\|U_{T U}\right\|=1\right)$.

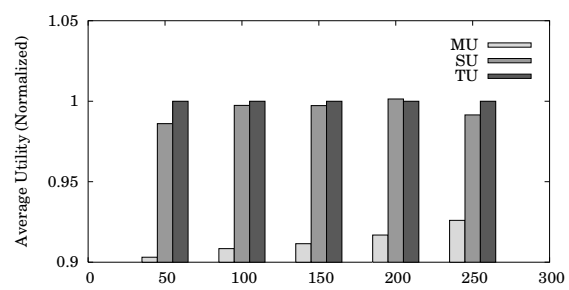

Number of Soft Tasks
(a) 150 hard tasks

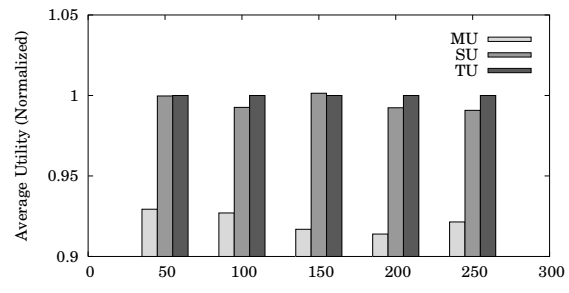

(b) 200 soft tasks

Fig. 11. Comparison among the heuristics (500 tasks)

From the extensive set of experiments that we have performed and its results (Figs. 9 through 11) we conclude, that if one of the proposed heuristics is to be chosen, TotalUTILITY is the procedure that should be used for solving the problem of scheduling with soft and hard tasks to maximize utility as formulated in Section 4. This does not mean that TotalUtility gives the best results in every case, but in average it performs better. Since the proposed heuristics are computationally cheap, we could run all three and choose, among their results, the schedule that yields the highest total utility.

In Section 6 we pointed out that the worst-case time complexity of the algorithms MaxUtility and SingleUTILITY is $O\left(|T|^{3}(|H|+|S|)\right)$ and that one of TotalUtiLity is $O\left(|T|^{3}\left(|H|+|S|^{2}\right)\right.$ ). In order to give a quantitative idea of the execution times of these heuristics and the exact algorithm we used throughout this section, we present in Table 1 the average running time for these algorithms in the case of systems containing 100 tasks out of which 50 are hard.

\begin{tabular}{|c|c|c|c|c|}
\hline \multirow{2}{*}{$\begin{array}{c}\text { Num. Soft } \\
\text { Tasks }\end{array}$} & \multicolumn{4}{|c|}{ Average Execution Time $[\mathrm{s}]$} \\
\cline { 2 - 5 } & Exact & MU & SU & TU \\
\hline 2 & 0.085 & 0.051 & 0.051 & 0.052 \\
\hline 3 & 0.237 & 0.053 & 0.052 & 0.053 \\
\hline 4 & 0.879 & 0.053 & 0.053 & 0.055 \\
\hline 5 & 3.623 & 0.055 & 0.055 & 0.058 \\
\hline 6 & 20.78 & 0.056 & 0.056 & 0.059 \\
\hline 7 & 115.36 & 0.057 & 0.058 & 0.061 \\
\hline 8 & 896.36 & 0.059 & 0.059 & 0.063 \\
\hline
\end{tabular}

Table 1. Av. execution times (100 tasks, 50 hard tasks)

\section{Conclusions}

We have presented an approach to the problem of static scheduling of real-time systems that have hard and soft tasks.
Our approach considers that hard, soft, and non-real-time tasks are periodic and they all are mapped into a single processor.

We made use of non-increasing utility functions to represent the relevance of soft tasks and how the quality of results is diminished when missing a soft deadline. The problem we have addressed is thus that one of finding the execution order of tasks in such a way that the sum of individual utilities of soft tasks is maximum and, at the same time, there is guarantee that no hard deadline will be missed. We used maximum duration of tasks for guaranteeing hard deadlines and expected duration of tasks for calculating the total utility.

We have proposed an exact algorithm for solving the problem of static scheduling with soft and hard tasks to maximize utility, which gives the optimal schedule in $O\left(|T|^{3}|H||S| !\right)$ time. We also presented three heuristic procedures that find near-optimal solutions in short time.

We have randomly generated 15000 task graphs for experimental evaluation. The experiments showed that the heuristic TOTALUTILITY is the one that gives the best results in average. Its time complexity $\left(O\left(|T|^{3}\left(|H|+|S|^{2}\right)\right)\right)$ is however larger than the one of the other two heuristics $\left(O\left(|T|^{3}(|H|+|S|)\right)\right)$. In the cases where it was feasible to compute the optimal schedule (up to 8 soft tasks), we obtained an average deviation smaller than $2 \%$ when using the heuristic TU.

As part of our future work, we are currently studying the issue of quasi-static scheduling of real-time systems with soft and hard tasks. The idea is to prepare at design-time a number of schedules and schedule-switching points, and let the decision of which of them to follow be taken at runtime based on the actual execution times. Thus we can further improve the quality of results (in terms of total utility) by choosing a schedule that best fits the actual conditions without incurring the expensive on-line computation of such schedules.

\section{References}

[1] L. Abeni and G. Buttazzo. Integrating Multimedia Applications in Hard Real-Time Systems. In Proc. Real-Time Systems Symposium, pages 4-13, 1998.

[2] G. Buttazzo and F. Sensini. Optimal Deadline Assignment for Scheduling Soft Aperiodic Tasks in Hard Real-Time Environments. IEEE. Trans. on Computers, 48(10):1035-1052, Oct. 1999.

[3] L. A. Cortés, P. Eles, and Z. Peng. Static Scheduling of Monoprocessor Real-Time Systems composed of Hard and Soft Tasks. Technical report, Embedded Systems Lab, Dept. of Computer and Information Science, Linköping University, Linköping, Sweden, Apr. 2003.

[4] R. I. Davis, K. W. Tindell, and A. Burns. Scheduling Slack Time in Fixed Priority Pre-emptive Systems. In Proc. RealTime Systems Symposium, pages 222-231, 1993.

[5] H. Kaneko, J. A. Stankovic, S. Sen, and K. Ramamritham. Integrated Scheduling of Multimedia and Hard Real-Time Tasks. In Proc. Real-Time Systems Symposium, pages 206217, 1996.

[6] J. P. Lehoczky and S. Ramos-Thuel. An Optimal Algorithm for Scheduling Soft-Aperiodic Tasks in Fixed-Priority Preemptive Systems. In Proc. Real-Time Systems Symposium, pages 110-123, 1992.

[7] C. D. Locke. Best-Effort Decision Making for Real-Time Scheduling. PhD thesis, Department of Computer Science, Carnegie-Mellon University, Pittsburgh, May 1986.

[8] I. Ripoll, A. Crespo, and A. García-Fornes. An Optimal Algorithm for Scheduling Soft Aperiodic Tasks in DynamicPriority Preemptive Systems. IEEE. Trans. on Software Engineering, 23(6):388-400, Oct. 1997. 